$H$ pylori

\title{
Experimental Helicobacter pylori infection in humans: a multifaceted challenge
}

\section{P Michetti}

\section{Is there a scientific rationale for the use of an infection challenge model for Helicobacter pylori vaccine development in humans?}

C hallenge experiments have been an important method of studying the pathogenesis of many infectious diseases and of evaluating initial efficacy of vaccines before large scale field tests are conducted. ${ }^{12}$ In challenge experiments, infections are deliberately induced under carefully controlled and monitored conditions to healthy research volunteers. Induced infections are usually either self limiting or can be fully treated within a short period of time. Because physicians should be dedicated to alleviating disease and avoiding harm to patients, this type of experiment may cause uncomfortable symptoms and evoke serious moral concerns. It should be appreciated however that clinical research commonly involves risks to subjects that are not outweighed by medical benefits but are justified by the potential to acquire new knowledge. ${ }^{3}$ In that regard, infection inducing challenges are not necessarily more ethically problematic than phase I trials aimed at determining maximum tolerated doses of medications. Like any clinical research, challenge experiments should be conducted by competent investigators according to sound protocols that incorporate appropriate safeguards to ensure the safety of volunteers. Because these experiments may provide valuable information that might not be otherwise obtained, lead to novel therapies, or speed up vaccine development that will ultimately spare morbidity or death from infectious diseases and reduce exposure of large groups in field trials, challenge experiments may be justified. ${ }^{4}$ However, the scientific rationale should be carefully examined for any given pathogen and model. When such a rationale exists, then the question of risks and discomforts should be addressed.

Twenty years after the first culture of Helicobacter pylori there are still many gaps in our knowledge of this worldwide infection. In particular, little information is available regarding the precise mode of transmission of the infection, the conditions required for exposure to $H$ pylori to lead to chronic gastric infection, and the early clinical and pathological events following colonisation of the gastric mucosa. ${ }^{5}$ Indeed, the infection is typically acquired in childhood but most studies related to $H$ pylori pathogenesis and host response are conducted, for obvious ethical and practical reasons, in adults. As young children are not always chronically infected following initial infection, ${ }^{6}$ a better understanding of the early phase of the infection may prove useful in the development of novel therapies and vaccines. Although it is not entirely clear that the early events in $H$ pylori infection in adults are identical to those in children during natural infection, adult infection has been reported and has led to typical chronic infection. ${ }^{7}$ A $H$ pylori challenge model in adults may thus present an unique opportunity to obtain information on the pathogenesis of this bacterium, which may be potentially useful in the development of new drugs or obtaining knowledge on the host response crucial for vaccine development.

By inducing experimental $H$ pylori infection in healthy volunteers, the report of Graham and colleagues ${ }^{8}$ in this issue of Gut has certainly contributed to our understanding of this infection (see page 1235). This study provides valuable information on the low inoculum size required for infection, the impact of some virulence factors, the symptoms associated with acute $H$ pylori infection, the physiological changes induced by $H$ pylori, as well as on the rapid development of histological changes previously thought to be linked to chronic infection. This harvest of valuable information would have been very difficult to obtain by other means, especially if early infection is to be analysed in children. These data represent a well controlled body of information, useful to the scientific community, to better understand transmission and to further validate animal models for pathogenesis studies.

In addition to its use in studying the pathogenesis of infectious diseases, infection inducing challenge experiments have been used to evaluate the initial efficacy of vaccines before conducting large scale field tests for many infectious diseases, including enteric pathogens. ${ }^{9}$ Typically, this step is undertaken after basic research has provided data regarding potential protective antigens, and allowed for a description of the host immune response. Then, ideally, animal models that mimic human infection and response are used to test efficacy before human studies are considered. Finally, candidate vaccine preparations should then be evaluated for safety and immunogenicity in humans, outside of the challenge setting, to minimise exposure of volunteers only to the most promising candidates. In this instance, however, it is of central importance to determine whether the proposed human challenge model is not only suited to reproduce the natural infection but also corresponds to the population that the vaccine is intended to protect.

Vaccine development against $H$ pylori started over 10 years ago, after a proof of principle was established in mice infected with Helicobacter felis. ${ }^{10}{ }^{11}$ In this model, and later in $H$ pylori mouse models, several protective antigens of $H$ pylori were identified. Urease was also shown to be effective in a vaccine preparation administered to infected animals. ${ }^{12}$ Urease, cytotoxin associated gene A (CagA), vacuolating cytotoxin (VacA), and $H$ pylori neutrophil activating protein (HpNAP) were also shown to be safe and immunogenic in humans once their protective potential had been established in mice. ${ }^{13}$ Although these antigens are protective in mice, and elicit both humoral and cellular immune responses, no clear immune correlate of protection could be identified in mice, which prevents the use of an immunological test to evaluate the protective potential of a candidate vaccine in humans. ${ }^{14}$ Field trials thus represent the alternative, with protection as the primary outcome. As $H$ pylori infection is acquired early in life, this would imply that field trials would have to be conducted in children, in areas of high incidence (that is, on the less favoured children of most societies). If a prophylactic vaccine is to be developed, such a field approach would certainly elicit several serious ethical concerns. These concerns may be progressively addressed if benefit can be shown for children but the incentive to invest in the long research needed to follow that approach would certainly be helped by positive results in adults. As natural 
infection is rare in adults, a challenge model would then be required to progress substantially towards prophylactic vaccine development. In this context, the model developed by Graham and colleagues $^{8}$ certainly represents an advance. The value of this adult model in predicting vaccine induced protection in children however would further depend on the assumptions that both infection and vaccine induced protective responses are similar in adults and children. In addition, the model would not be appropriate for testing the protective potential of CagA, as the strain was selected as CagA negative for safety reasons. As virulence factors often represent important vaccine antigens, this is certainly a limitation of the model which could be resolved only after extensive safety data are obtained with the challenge model. When the goal is ultimately to develop a therapeutic vaccine however, testing could be conducted by vaccinating adults naturally infected with $H$ pylori. ${ }^{15}$ As far as vaccine development against $H$ pylori is considered, it is not yet clearly established that an infection inducing challenge model is likely to facilitate or speed up vaccine development.

In exploring the ethical justification of particular infection inducing challenge experiments, the nature of the infection has to be considered. Legitimate challenge experiments may include infection likely to produce mild symptoms not interfering with the subject's daily activities and those that are self limiting or that can be adequately eradicated with certainty. ${ }^{3}$ From the reports of the two investigators that infected themselves with $H$ pylori and from iatrogenic infection reports, it was not expected that acute $H$ pylori would cause more than mild dyspeptic symptoms. To further minimise this risk, the strain was obtained from a patient with no alarming symptom, histological changes, or complications of infection. The primary concern however was that experimental $H$ pylori infection may become chronic, and that a treatment regimen with $100 \%$ effectiveness to eradicate the infection was not available. ${ }^{16}$ Careful attention was paid by the investigators to selection of the challenge strain. It was derived from a patient that was eradicated without difficulty, and the strain tested for its lack of resistance to the antibiotics commonly used to treat the infection. However, it was CagA negative and the lack of this virulence factor had been associated with decreased eradication rates. Despite this, full confidence that $H$ pylori infection, even with a fully antibiotic sensitive strain, can be eradicated is lacking. Volunteers that might remain infected would then be at increased risk, albeit a small risk, of morbidity. In addition, as the precise transmission mode of $H$ pylori is as yet unclear, there was a small risk that the experimental strain would pass to other individuals. In the course of their study, Graham et al achieved 100\% eradication rate, and no apparent transmission of the infection was observed, possibly related to the precautions undertaken to minimise this risk. The size of the experiment however was far too small to ascertain that the strain can be safely eradicated and is not transmitted, with type II errors for these observations being possible. Therefore, the challenge model can not be considered safe, but as progress is made towards the control of $H$ pylori infection, the threshold for acceptability of this model, as for other infections, is likely to shift.

After the experiment had started, an expert panel assembled on 30 November 2000 by the National Institute for Health, Division of Microbiology and Infectious Diseases, concluded that the development of the $H$ pylori infection challenge model should not be pursued at this time. ${ }^{17}$ In brief, the reasons for this conclusion were that ( 1 ) there was no compelling evidence for the need for a Helicobacter vaccine in the USA, (2) no clear vaccination strategy was defined for the USA, (3) volunteers were at risk of unsuccessful eradication with possible late consequences, (4) animals models had not been fully investigated for vaccine efficacy, and (5) more conventional field trials were available to measure vaccine efficacy. These conclusions however may underestimate the full extent of the $H$ pylori problem worldwide and do not address the scientific rationale for the use of a challenge model for Helicobacter vaccine development. The $H$ pylori challenge experiment reported by Graham et al was conducted under the best available conditions and resulted in minimal harm to volunteers. The results shed light on interesting aspects of the pathogenesis of the bacteria, and provide validation information for the development of animal models. However, there are many more questions to be answered before this model can be validated and proven useful for vaccine development. The scientific community therefore needs to think carefully before deciding to use this model in humans for the development of a vaccine against $H$ pylori.

Gut 2004;53:1220-1221.

doi: 10.1136/gut.2004.042135

Correspondence to: Professor P Michetti, Division of Gastroenterology and Hepatology, BH10N-545, Centre Hospitalier Universitaire Vaudois, CH-1011 Lausanne, Switzerland; pierre.michetti@hospvd.ch

\section{REFERENCES}

1 Sack DA, Tacket CO, Cohen MB, et al. Validation of a volunteer model of cholera with frozen bacteria as the challenge. Infect Immun 1998;66:1968-72

2 Tacket CO, Binion SB, Bostwick $E$, et al. Efficacy of bovine milk immunoglobulin concentrate in preventing illness after Shigella flexneri challenge. Am J Trop Med Hyg 1992;47:276-83.

3 Miller FG, Grady C. The ethical challenge of infection-inducing challenge experiments. Clin Infect Dis 2001;33:1028-33.

4 Marwick C. Volunteers in typhoid infection study will aid future vaccine development. JAMA 1998;279: 1423-4.

5 Suerbaum S, Michetti P. Helicobacter pylori infection. N Engl J Med 2002;347: 1175-86.

6 Rowland M, Kumar D, Daly L, et al. Low rates of Helicobacter pylori reinfection in children. Gastroenterology 1999;117:336-41

7 Graham DY, Alpert LC, Smith JL, et al. latrogenic Campylobacter pylori infection is a cause of epidemic achlorhydria. Am J Gastroenterol 1988:83:974-80.

8 Graham DY, Opekun AR, Osato MS, et al. Challenge model for Helicobacter pylori infection in human volunteers. Gut 2004;53:1235-43.

9 Tacket CO, Kotloff KL, Losonsky G, et al. Volunteer studies investigating the safety and efficacy of live oral El Tor Vibrio cholerae O1 vaccine strain CVD 111. Am J Trop Med Hyg 1997:56:533-7.

10 Czinn SJ, Cai A, Nedrud JG. Protection of germfree mice from infection by Helicobacter felis after active oral or passive $\lg \mathrm{A}$ immunization. Vaccine 1993; 11:637-42.

11 Chen M, Lee A, Hazell S. Immunisation against gastric helicobacter infection in a mouse/ Helicobacter felis model. Lancet 1992;339:1120-1.

12 Corthésy-Theulaz I, Porta N, Glauser M, et al. Oral immunization with Helicobacter pylori urease $B$ subunit as a treatment against Helicobacter infection in mice. Gastroenterology 1995; 109:1 15-21.

13 Keller WC, Michetti P. Vaccination against Helicobacter pylori-an old companion of man. 2001; 1:795-802.

14 Ruggiero P, Peppoloni S, Rappuoli R, et al. The quest for a vaccine against Helicobacter pylori: how to move from mouse to man? Microbes Infect 2003;5:749-56.

15 Michetti P, Kreiss C, Kotloff KL, et al. Oral immunization with urease and Escherichia coli heat-labile enterotoxin is safe and immunogenic in Helicobacter pylori-infected adults. Gastroenterology 1999;1 16:804-12.

16 Perri F, Qasim A, Marras L, et al. Treatment of Helicobacter pylori infection. Helicobacter 2003;8(suppl 1):53-60.

17 Blaser MJ, Lang D. NIAID Workshop: Development of a Standardized Human Challenge Model for Helicobacter pylori, 1999. http://www.niaid.nih.gov/dmid/meetings/ HpChallengeMdl.htm. 


\section{Endoscopic mucosal resection: an evolving therapeutic strategy for non- polypoid colorectal neoplasia}

\section{R Kiesslich, M F Neurath}

\section{Endoscopic management for laterally spreading tumours of the colorectum is a safe and effective treatment and may be an alternative to surgery in selected patients}

C olorectal cancer develops in approximately $5-6 \%$ of the adult population and is one of the leading causes of cancer death in Europe and the USA. ${ }^{12}$ Screening colonoscopy is the widely accepted gold standard for early diagnosis of colorectal cancer and should be offered to patients older than 50 years. ${ }^{3-5}$ However, colonoscopy is increasingly in competition with computed tomographic virtual endoscopy. ${ }^{6}$ Therefore, it is essential to understand whether total colonoscopy rather than virtual endoscopy enables the detection of all relevant premalignant and malignant lesions as only early diagnosis of neoplasias offers the possibility for curative endoscopic or surgical resection.

Polypoid lesions are easy to detect by endoscopy. In contrast, non-polypoid lesions are often overlooked. ${ }^{7}$ The endoscopist must be aware of flat and depressed lesions because the primarily visible mucosal changes of such lesions are often very discrete. In this context, the endoscopist should look for slight colour changes, interruption of the capillary network pattern, slight deformation of the colonic wall, spontaneously bleeding spots, shape changes of the lesion with insufflation or deflation of air, and interruption of the innominate grooves. ${ }^{7}$

An important diagnostic tool for detection of non-polypoid colorectal lesions consists of chromoendoscopy. ${ }^{8}$ In contrast with ulcerative colitis where pan-chromoendoscopy is favourable, targeted staining with indigo carmine or methylene blue in a selective fashion is sufficient for non-polypoid colorectal lesions. ${ }^{10}$ Chromoendoscopy helps to unmask such lesions in the colon and to delineate its borders. By the help of magnifying endoscope, the stained surface and crypt architecture can be analysed, and differentiation between non-neoplastic and neoplastic lesions becomes possible with high accuracy. ${ }^{11}$
Recently, it was shown in a prospective randomised trial that magnifying endoscopy is superior to standard videoendoscopy because of the possibility of analysing stained colorectal lesions at higher magnifications. ${ }^{12}$ Differentiation between non-neoplastic and neoplastic lesions was possible however when using high magnification chromoendoscopy to differentiate between noninvasive and invasive neoplasias but sensitivity decreased to $50 \%{ }^{10}$

Initially, knowledge about non-polypoid colorectal lesions was based on observations in Japanese patients. ${ }^{16}$ Similar observations have since been made in Western populations using magnifying chromoendoscopy. In fact, introduction of newly developed magnifying and high resolution endoscopes, as well as the renaissance of chromoendoscopy, have led to a significant increase in the detection of non-polypoid lesions in the Western world. In recent prospective chromoendoscopic studies, $7-31 \%$ of diagnosed lesions were flat or depressed..$^{813-15}$ Furthermore, Saitoh and colleagues $^{8}$ observed that $62 \%$ of flat and depressed lesions could be judged correctly by the help of intravital staining. Thus Western endoscopists have to recognise these newly discovered lesions. For this reason, it is essential to discuss the malignant potential, screening, and therapeutic strategies of such non-polypoid lesions.

Macroscopic appearance of colorectal lesions can be classified according to the Japanese classification of colorectal cancer (see fig 1). ${ }^{17}$ However, Kudo et al have recommended a more practical classification. ${ }^{7}$ Here, early non-polypoid colorectal neoplasias can be classified as slightly elevated (small flat adenoma $<\mathrm{lcm}$ ), laterally spreading tumours (large flat adenoma $>1 \mathrm{~cm}$ ) or depressed (see fig 2). Specifically, laterally spreading tumours (LST) of the colorectum are defined as lesions of more than $10 \mathrm{~mm}$ in diameter with a low vertical axis which extends laterally along the interior luminal wall. Such LSTs can be further subdivided into granular and non-granular types. ${ }^{7}$

In this issue of Gut, Hurlstone and colleagues $^{18}$ report their experience with colonoscopic resection of 82 LSTs of the colorectum (see page 1334). Firstly, identification of these lesions was made with the help of dye spraying, underlining the high impact of targeted chromoendoscopy during screening colonoscopy. Using this approach, a total of 26 non-granular and 56 granular LSTs were identified. The non-granular type of LSTs was more often associated with invasive disease and was frequently localised to the right colon.

The theory of the adenoma-carcinoma sequence is well established for polypoid adenomas. ${ }^{19}$ Some authors have advocated a new theory of cancer development with special attention to the superficial forms of cancer that exhibit non-polypoid growth. They postulated a higher risk of malignant invasion compared with polypoid neoplasms.. ${ }^{21}$ Hurlstone and colleagues ${ }^{15}$ observed areas of high grade dysplasia in $25 \%$ of flat adenomas whereas high grade dysplasia was observed in only $12 \%$ of protuberant adenomas, consistent with the idea that such flat lesions have a high risk for malignancy. However, previous studies suggested that among non-polypoid lesions, only depressed lesions have a higher risk of malignant infiltration. Up to a size of $2 \mathrm{~cm}$, the rate of malignant infiltration is $40 \%$ for depressed lesions compared with only $1.3 \%$ for polypoid lesions. ${ }^{7}$ Surprisingly, the rate of lymph node metastasis is similar for polypoid (11\%) and nonpolypoid lesions (11\%). Even for depressed lesions with the tendency to grow towards the muscularis propria, the rate of lymph node metastasis has been reported as only $6-7 \% .^{22}$

Early colorectal carcinoma is defined by its depth of infiltration (the carcinoma is invading the submucosal layer). The degree of submucosal invasion is classified into three types based on depth of invasion: when less than one third of the submucosa is invaded the stage is called sml and if more than two thirds are invaded the stage is sm3. Stage $\operatorname{sm} 2$ is intermediate. Sml early colorectal cancers without lymphatic or venous invasion have almost no risk of lymph node metastasis. ${ }^{7}$ Thus they can be removed endoscopically.

Severe dysplastic epithelium confined to the mucosa is called severe dysplasia or intramucosal carcinoma, although there is some controversy as to the terminology for this particular lesion. Japanese pathologists tend to use the term "intramucosal or mucosal 


Not necessary $\left\{\begin{array}{c}\text { Protruded } \\ \text { type } \\ \text { Highly }\end{array}\right.$

Figure 1 Diagnostic classification ${ }^{17}$ and malignant potential of early colorectal cancer.

carcinoma" whereas Western pathologists tend to avoid this term to prevent unnecessary major surgery. However, these lesions are ideally suited for endoscopic resection. ${ }^{22}$

Selection of suitable lesions for endoscopic resection is of crucial clinical importance. Hurlstone et al have developed essential inclusion and exclusion criteria. ${ }^{18}$ According to these criteria, 56 of 82 identified LSTs underwent endoscopic mucosal resection and high "cure" rates were achieved. The results show impressively that endoscopic management for LST is possible and justified. However, the question remains how the endoscopic or surgical management of non-polypoid lesions should be performed? Depressed lesions are often malignant and have been shown to rapidly invade the submucosal layer.
Therefore, this group of lesions should always be treated. Those confined to the mucosa or that only slightly invade the submucosa (without vessel infiltration) can be completely removed and cured by endoscopic resection. If histology shows that the cancer has massively invaded the submucosa or permeates the vessels, additional surgery is required.

Signs of advanced carcinoma are depressed lesions $>10 \mathrm{~cm}$ and unstructured crypt architecture after staining, as well as lacking the lifting sign after saline injection. These lesions should undergo surgery primarily.

Flat lesions up to a size of $5 \mathrm{~mm}$ without suspected staining pattern or depression can be removed by biopsy. Lesions up to $2 \mathrm{~cm}$ should be resected en bloc whereas lesions larger than $2 \mathrm{~cm}$ can be treated by peacemeal

\begin{tabular}{|c|c|c|c|}
\hline Type & Macroscopic appearance & Size $(\mathrm{cm})$ & Recommended therapy \\
\hline \multirow{2}{*}{$\begin{array}{l}\text { Slightly elevated type } \\
\text { (flat adenoma) }\end{array}$} & & $<0.5$ & Biopsy \\
\hline & & $0.5-1.0$ & Mucosal resection \\
\hline
\end{tabular}

Laterally spreading type

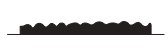

1.0-2.0 Mucosal resection, en bloc

$>2.0 \quad$ Mucosal resection, peace meal

\begin{tabular}{lll} 
Depressed type & $<1.0$ & Mucosal resection, en bloc \\
\hdashline-1.0 & Surgery
\end{tabular}

Prerequisite for endoscopic therapy: neoplastic straining pattern, positive lifting sign after saline injection, maximum diameter $<1 / 3$ of the luminal circumference.

Figure 2 Classification of non-polypoid colorectal lesions ${ }^{7}$ and recommended therapy. resection. ${ }^{718}$ Peacemeal resection harbours the risk of local recurrence, as shown by Hurlstone and colleagues, ${ }^{18}$ and an intense follow up programme should therefore be added (see fig 2).

Complete resection should always be intended. However, chromoendoscopy after resection helps to identify remnant islands of neoplastic tissue which can be removed by argon beam coagulation. ${ }^{18}{ }^{23}$ Hurlstone and colleagues ${ }^{18}$ reported a local recurrence rate of $17 \%$ and recurrent disease in $10 \%$. In patients with local recurrence, eight of 10 underwent further endoscopic resection without residual disease present at 24 months of follow up. These data are comparable with previous Japanese experience. ${ }^{24} 25$ In the current study, Hurlstone and colleagues $^{18}$ thus showed that neither the diagnosis nor the treatment of nonpolypoid lesions is a Japanese phenomenon or expertise. Western endoscopists should be aware of these lesions and need to include mucosal resection for flat and depressed colorectal lesions into their therapeutic strategies.

In conclusion, introduction of magnifying endoscopy in combination with intravital staining has led to a significant improvement in the diagnosis of non-polypoid neoplasms. Flat and depressed lesions are now increasingly diagnosed in the Western world during colonoscopy using this new technique. In contrast, the diagnostic yield of alternative approaches such as computed tomography virtual endoscopy decreases for small lesions. ${ }^{6}{ }^{26}$ Differentiated knowledge about nonpolypoid lesions is essential to identify suitable lesions for endoscopic resection. Endoscopic mucosal resection is the treatment of choice for defined nonpolypoid lesions and offers a sufficient and curative therapy. Because of the possible higher malignant potency of non-polypoid lesions, an intense endoscopic follow up programme should be initiated and offers the possibility of repeat endoscopic treatment for local recurrence.

Gut 2004;53:1222-1224.

doi: $10.1136 /$ gut.2004.043281

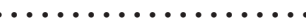

Authors' affiliations

R Kiesslich, M F Neurath, I Med Clinic, Johannes Gutenberg University Mainz, Germany

Correspondence to: Dr R Kiesslich, I Med Klinik und Poliklinik, Johannes Gutenberg Universität Mainz, Langenbeckstr 155101 Mainz,

Germany; info@ralf-kiesslich.de

\section{REFERENCES}

1 Grady WM. Genetic testing for high-risk colon cancer patients. Gastroenterology 2003;124:1574-94. 
2 Burt RW. Colon cancer screening Gastroenterology 2000; 1 19:837-53.

3 Gatta G, Ciccolallo L, Capocaccia R, et al. Differences in colorectal cancer survival between European and US populations: the importance of sub-site and morphology. Eur J Cancer 2003;39:2214-22.

4 Weir HK, Thun MJ, Hankey BF, et al. Annual report to the nation on the status of cancer, 1975 2000, featuring the uses of surveillance data for cancer prevention and control. J Natl Cancer Inst 2003;95: 1276-99.

5 Winawer S, Fletcher R, Rex D, et al. Colorectal cancer screening and surveillance: clinical guidelines and rationale-Update based on new evidence. Gastroenterology 2003; 124:544-60.

6 Pickhardt PJ, Choi JR, Hwang I, et al. Computed tomographic virtual colonoscopy to screen for colorectal neoplasia in asymptomatic adults. N Engl J Med 2003;349:2191-200.

7 Kudo S, Kashida H, Tamura T, et al. Colonoscopic diagnosis and management of nonpolypoid colorectal cancer. World J Surg 2000:24: 1081-90.

8 Saitoh Y, Waxman I, West AB, et al. Prevalence and distinctive biologic features of flat colorectal adenomas in a North American population. Gastroenterology 2001;120:1657-65.

9 Kiesslich RJ, Fritsch $M$, Holtmann $M$, et al. Methylene blue-aided chromoendoscopy for the detection of intraepithelial neoplasia and colon cancer in ulcerative colitis. Gastroenterology 2003; 124:880-8.

10 Hurlstone DP, Cross SS, Adam I, et al. Efficacy of high magnification chromoscopic colonoscopy for the diagnosis of neoplasia in flat and depressed lesions of the colorectum: a prospective analysis. Gut 2004;53:284-90.

11 Kudo S, Tamura S, Nakajima T, et al. Diagnosis of colorectal tumorous lesions by magnifying endoscopy. Gastrointest Endosc 1996;44:8-14

12 Konishi K, Kaneko K, Kurahashi T, et al. A comparison of magnifying and nonmagnifying colonoscopy for diagnosis of colorectal polyps: A prospective study. Gastrointest Endosc 2003;57:48-53.

13 Rembacken BJ, Fujii T, Cairns A, et al. Flat and depressed colonic neoplasms: a prospective study of 1000 colonoscopies in the UK. Lancet 2000:8:1211-14

14 Kiesslich $\mathbf{R}$, von Bergh $M$, Hahn $M$, et al. Chromoendoscopy with indigocarmine improves the detection of adenomatous and

nonadenomatous lesions in the colon. Endoscopy 2001;33:1001-6.

15 Hurlstone DP, Cross SS, Adam I, et al. A prospective clinicopathological and endoscopic evaluation of flat and depressed colorectal lesions in the United Kingdom. Am J Gastroenterol 2003;98:2543-9.

16 Matsui T, Yao T, Iwashita A. Natural history of colorectal cancer. World J Surg 2000;24:1022-8.

17 Japanese Research Society for Cancer of the Colon and Rectum. General rules for clinical and pathological studies on cancer of the colon, rectum and anus. Part II. Histological classification. Jpn J Surg 1983;23:574

18 Hurlstone DP, Sanders DS, Cross SS, et al. Colonoscopic resection of lateral spreading tumours: a prospective analysis of endoscopic mucosal resection. Gut 2004;53:1334-9.

19 Muto T, Bussey HJR, Morson BC. The evaluation of cancer of the colon and rectum. Cancer 1975; $36: 2251$

20 Kuramoto S, Oohara T. Flat early cancers of the large intestine. Cancer 1989;15:950-5.

21 Minamoto T, Sawaguchi K, Ohta T, et al. Superficial-type adenomas and adenocarcinomas of the colon and rectum: a comparative morphological study. Gastroenterology 1994; 106:1436-43.

22 Ajioka Y, Watanabe H, Kazama S, et al. Early colorectal cancer with special reference to the superficial nonpolypoid type from a histopathologic point of view. World J Surg 2000;24:1075-80

23 Brooker JC, Saunders BP, Shah SG, et al. Treatment with argon plasma coagulation reduces recurrence after piecemeal resection of large sessile colonic polyps: a randomized trial and recommendations. Gastrointest Endosc 2002;55:371-5.

24 Tanaka S, Haruma K, Oka S, et al. Clinicopathologic features and endoscopic treatment of superficially spreading colorectal neoplasms larger than $20 \mathrm{~mm}$. Gastrointest Endosc 2001 ;54:62-6.

25 Tamura S, Nakajo K, Yokoyama Y, et al. Evaluation of endoscopic mucosal resection for laterally spreading rectal tumors. Endoscopy 2004;36:306-12.

26 Laghi A, lannaccone R, Carbone I, et al. Detection of colorectal lesions with virtual computed tomographic colonography. Am J Surg 2002; 183:124-31

\section{Diagnostic biopsy for hepatocellular carcinoma in cirrhosis: useful, neces- sary, dangerous, or academic sport?}

\author{
J Schölmerich, D Schacherer
}

At present, in experienced hands, ultrasound guided biopsy using either cut needles or fine needle aspiration, seems to be technically appealing and most effective for diagnosis of hepatocellular carcinoma

$\mathrm{H}$ epatocellular carcinoma (HCC) is the eighth most frequent tumour entity worldwide (one million per year). The incidence in the USA and elsewhere has increased, which is probably due to the rise in the incidence of hepatitis $\mathrm{C}$ virus infection. A number of cohort studies have shown that surveillance using abdominal ultrasound (US) improves management of HCC developing in cirrhotic patients as it allows early detection and application of potentially curative treatments. ${ }^{1}$ Thus far it is not entirely clear whether surveillance increases survival as no randomised controlled trials have been conducted to answer this question. However, as eventually curative treatments such as resection, ablation, and in particular liver transplantation have emerged as effective means of treating HCC as long as it is not too large and too widespread, early diagnosis seems to be a reasonable goal in improving the poor prognosis of this cancer. $^{2-4}$ Interestingly, it has recently been shown by comparison of time periods that besides being part of the more recent cohort, tumour staging was the only independent predictor of survival. ${ }^{1}$ Thus it seems obvious that detection of early carcinoma in liver cirrhosis would be a reasonable goal. Progress made in recent years regarding imaging, in particular spiral computed tomography and using the increased vascularity of HCC, allowing for better scanning protocols, has increased the detection rate significantly. In addition, magnetic resonance imaging has become the diagnostic procedure of choice in some institutions. However, most experts still advocate liver biopsy. ${ }^{5-11}$

In addition, improved US techniques such as contrast enhancement with and without Doppler ultrasonography ${ }^{12} 13$ may improve early diagnosis further. However, no adequate comparisons between all competing techniques are available to date. Most studies have used two or a maximum of three modalities and have come to different conclusions. ${ }^{14}$ The role of positron emission tomography in different modes is also not clear but it does not seem to be very helpful. ${ }^{15}$ Well performed large studies on modalities for early diagnosis of HCC are therefore welcome.

In this issue of Gut, a study from Italy $^{16}$ reports on 4375 patients with newly detected cirrhosis and no focal lesions at initial US followed over a period of nine years (see page 1356). Of 4581 patients agreeing to participate, 206 had nodular lesions at initial US. Patients were followed every 4-6 months using $\alpha$ fetoprotein (AFP) and abdominal US. If a lesion was diagnosed and AFP levels were above $400 \mathrm{ng} / \mathrm{ml}$, HCC was assumed and treated accordingly. Lesions associated with an AFP level below $400 \mathrm{ng} / \mathrm{ml}$ underwent US guided fine needle biopsy (FNB) performed by an experienced gastroenterologist using fine cutting needles and non-cutting spinal needles. Choice between needles was left to the individual examiner. When histology or cytology indicated HCC, the patient was treated. If biopsy revealed a negative result, computed tomography and, in one centre, contrast enhanced magnetic resonance imaging were performed. A total of 688 new focal lesions of 6-42 $\mathrm{mm}$ in diameter were detected. In 294 patients (43\%), lesions were 


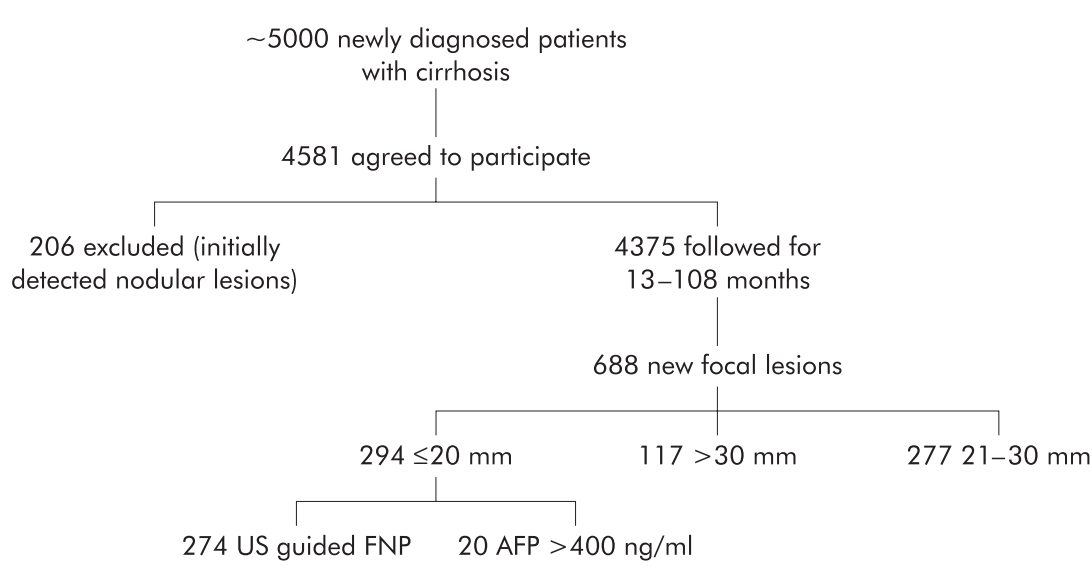

Figure 1 Overview of the study. ${ }^{16}$ AFP, $\alpha$ fetoprotein; US, ultrasound; FNP, fine needle biopsy.

$\leqslant 20 \mathrm{~mm} ; 258$ of these $(87.6 \%)$ were diagnosed as having HCC. In those with very small nodules $(\leqslant 10 \mathrm{~mm})$, HCC was diagnosed in 69\% of cases in contrast with $91 \%$ in those with nodules of 11-20 mm. Only 20 of 294 patients had an elevated AFP $>400 \mathrm{ng} / \mathrm{ml}$. The remaining 274 underwent biopsy (fig 1). In 245 patients, a correct diagnosis was made which was confirmed by follow up: 210 patients had HCC, four had a non-Hodgkin lymphoma, and 31 were found to be benign, as judged by follow up. Twenty nine of 274 cases were false negatives. Thus in a screening population, more than $66 \%$ of small nodules arising in cirrhotic livers may prove to be HCC and $90 \%$ of those can be identified using US guided FNB. ${ }^{16}$

This topic has been discussed previously in this and other journals ${ }^{17-22}$ 15-20 years ago. Very similar results regarding the sensitivity of AFP and good sensitivity of US guided biopsy have been reported. ${ }^{21}$ A larger series conducted 10 years ago again revealed similar results. ${ }^{23}$

Why then do we need new studies and why do we still not know how to proceed? This may be due to the fact that three questions still remain unanswered:

(1) How good is the technique and which is the preferred technical modality to perform a biopsy?

(2) How dangerous is such a procedure and does it interfere with later treatment?

(3) Is a biopsy necessary at all, and does it change the outcome?

EFFICACY OF US GUIDED BIOPSY When considering the historical review given above and data from the paper published in this issue of Gut, $^{16}$ it appears at first glance that US guided biopsy has a very high efficacy in defining HCC. A rate of $10 \%$ false negatives is probably difficult to beat by any other technique. However, a recent study following 12 cases of lesions with an initial fine needle aspiration diagnosis of regenerating nodules revealed subsequently that 10 of these patients had a HCC. Thus a negative biopsy result is probably not helpful in suspected HCC, as in any other suspected tumour. If the initial biopsy is negative, repeat biopsy leads to diagnostic gain in one third of patients, in particular if the first biopsy provided a non-diagnostic sample (necrosis) or a false negative result due to well differentiated HCC. ${ }^{24}$ It is not entirely clear if there is a major difference between cutting and aspiration needles-early studies did not find a difference when an experienced pathologist was involved. ${ }^{25}$ The positive predictive value of imaging modalities has recently been shown to be rather high. ${ }^{11}$ However, this is not the case in all studies. A recent study from Japan demonstrated that many nodules detected by ultrasonography could not even be found by computed tomography. ${ }^{8}$ Another study used digital subtraction angiography and magnetic resonance imaging and found that imaging alone was sufficient to diagnose HCC in $58 \%$ of well differentiated and $87 \%$ of moderately and poorly differentiated nodules in 139 patients with chronic liver disease, where 207 nodules had been found by periodic surveillance. ${ }^{9}$ Imaging alone allowed for diagnosis of HCC in $60 \%$ of cases: $55 \%$ of patients with nodules of $10 \mathrm{~mm}$ in diameter or less required biopsy. With these conflicting results and the data of Caturelli and colleagues, ${ }^{16}$ it appears that at present, in experienced hands, US guided biopsy using either cut needles or fine needle aspiration seems to be technically appealing and most effective. However, the approach chosen by the authors does not necessitate biopsy in all patients as those patients with AFP levels $>400 \mathrm{ng} / \mathrm{ml}$ and those with typical features of HCC in larger nodules using imaging are taken for granted. Only 274 nodules in 4375 patients were biopsied because their size was $\leqslant 20 \mathrm{~mm}$ in diameter.

\section{COMPLICATIONS}

With regard to the second question, data are also contradictory. Overall, complications of US guided diagnostic and therapeutic procedures on focal liver lesions are rare. In a large series from the same authors, 16648 guided biopsies and 3035 therapeutic procedures in 13222 patients were analysed. Overall mortality was $0.06 \%$ (none after diagnostic procedures). ${ }^{26}$ However, the bleeding risk is greater in HCC as these lesions are usually hypervascular. In a series of 159 HCC patients undergoing fine needle aspiration, four $(2.5 \%)$ bled significantly, one of whom died. ${ }^{27}$ Thus bleeding has to be considered as a possible complication of biopsy in this tumour entity, in particular with subcapsular localisation of the tumour.

Another "complication" is the risk of mismanagement after interpretative errors. It is sometimes very difficult to distinguish well differentiated HCC from benign regenerative nodules, and criteria used by pathologists need to be very clear. ${ }^{28}$ With fine needle aspiration in particular, diagnosis may be difficult; aspiration of necrotic material seems to be another limiting factor for correct interpretation. ${ }^{10}$

Others consider needle tract seeding of malignant cells an important risk factor. This risk was found to be $5.1 \%$ (three patients) in a series of 59 patients. ${ }^{29}$ It was smaller in a series of 122 patients ( $1.6 \%$ (two patients)). ${ }^{30} \mathrm{No}$ recurrence was observed after local excision in both series. However, in other series the risk of seeding was much lower. ${ }^{31}$ These series were however the result of questionnaires, with all the uncertainties inherent in this approach.

In our view, there are risks present but these are negligible and, in particular, the risk of needle seeding should not preclude biopsy if the result influences treatment choice. The risk of seeding has to be weighted against a $2.5 \%$ unnecessary surgery rate when patients are not biopsied..$^{11}$ An exception may be patients with advanced liver cirrhosis who are awaiting transplantation, independent of a possible tumour. Here tumour seeding could be disastrous and a "wait and see strategy" may be reasonable. However, a biopsy may be helpful because in the case of a tumour, one would have to do an adequate "bridging therapy" until transplantation (for example, chemoembolisation) or consider early transplantation (for example, by a living donor). 


\section{NEED FOR BIOPSY}

The question of whether biopsy is necessary is also difficult to answer with the data available. Examining again the paper of Caturelli and colleagues ${ }^{16}$ it is obvious that in many patients an early diagnosis was made which may allow for different treatment options, such as percutaneous ethanol injection, resection, or liver transplantation. A recent study demonstrated that percutaneous ethanol injection had a similar survival rate as surgery and was actually approximately $60 \%$ at five years for patients with small HCC. ${ }^{32}$ However, the European Association for the Study of Liver currently recommends a "wait and see policy" for small lesions s $^{33}$ as only half of these are considered to be HCC. The current study contradicts this assumption. ${ }^{34}$ However, the question of whether obtaining a liver biopsy affects clinical outcome cannot yet be answered with the current information. Early detection of HCC seems to increase the chance of treatment, at least in Hong Kong, ${ }^{35}$ and also seems to improve prognosis, as recently described in this journal. $^{36}$ It is doubtful if randomised controlled studies will ever be conducted to answer this question definitively.

In the past, outcome for most patients, regardless of stage, was believed to be poor. However, as transplantation results in a prolonged disease free survival or even cure, as shown by some studies (for example, Mazzaferro and colleagues ${ }^{37}$ ), biopsy with needle seeding would actually deny the patient treatment and therefore change the clinical outcome significantly. Thus in our opinion, patients waiting for a transplant should only be biopsied if AFP levels are less than $20 \mathrm{ng} / \mathrm{ml}$, if the lesion cannot be characterised as HCC using other modalities (all of them should be performed), and if the presence of a HCC favourably alters the patient's candidacy for liver transplantation. If resection appears to be the best option, biopsy may or may not be performed. When palliative treatment is planned, biopsy makes sense in order to avoid unnecessary treatment.

In summary, the study of Caturelli and colleagues ${ }^{16}$ provides long sought after and important information on the possible efficacy of US guided biopsy of HCC in experienced hands and will probably define the gold standard of quality of this procedure. However, it does not answer the question of whether biopsy should be performed and, if so, in which patients when HCC is suspected on the basis of a cirrhotic liver. This remains to some extent a bedside decision, which probably should be made after discussion between surgeons, hepatologists, and interventionalists, and will be dependent on the treatment options available for the individual patient.

Gut 2004;53:1224-1226

doi: $10.1136 /$ gut.2004.040816

\section{Authors' affiliations}

J Schölmerich, D Schacherer, Department of Internal Medicine I, University Regensburg, Regensburg, Germany

Correspondence to: Professor J Schölmerich, Department of Internal Medicine I, University Regensburg, D-93042 Regensburg, Germany; juergen.schoelmerich@klinik.uni-r.de

\section{REFERENCES}

1 Sangiovanni A, Del Ninno E, Fasani $P$, et al. Increased survival of cirrhotic patients with a hepatocellular carcinoma detected during surveillance. Gastroenterology 2004; 126:1005-14.

2 Befeler AS, Di Bisceglie AM. Hepatocellular carcinoma: diagnosis and treatment. Gastroenterology 2002;122:1609-19.

3 Llovet JM, Bruix J. Systematic review of randomized trials for unresectable hepatocellular carcinoma: chemoembolization improves survival. Hepatology 2003;37:429-42.

4 Dick EA, Taylor-Robinson SD, Thomas HC, et al. Ablative therapy for liver tumours. Gut 2002;50:733-9.

5 Lencioni R, Cioni D, Crocetti L, et al. Magnetic resonance imaging of liver tumors. J Hepatol 2004:40:162-71.

6 Stoker J, Romijn MG, de Man RA, et al. Prospective comparative study of spiral computer tomography and magnetic resonance imaging for detection of hepatocellular carcinoma. Gut 2002;51:105-7.

7 Brancatelli G, Baron RL, Peterson MS, et al. Helical CT screening for hepatocellular carcinoma in patients with cirrhosis: frequency and causes of false-positive interpretation. AJR Am J Roentgenol 2003; 180:1007-14

8 Tanaka Y, Sasaki Y, Katayama K, et al. Probability of hepatocellular carcinoma of small hepatocellular nodules undetectable by computed tomography during arterial portography. Hepatology 2000;31:890-8.

9 Horigome $\mathrm{H}$, Nomura T, Saso K, et al. Limitations of imaging diagnosis for small hepatocellular carcinoma: comparison with histological finding. J Gastroenterol Hepatol 1999; 14:559-65.

10 Souto E, Gores GJ. When should a liver mass suspected of being a hepatocellular carcinoma be biopsied? Liver Transp/ 2000;6:73-5.

11 Torzilli G, Minagawa M, Takayama T, et al. Accurate preoperative evaluation of liver mass lesions without fine-needle biopsy. Hepatology 1999;30:889-93.

12 Fracanzani AL, Burdick L, Borzio $M$, et al. Contrast-enhanced doppler ultrasonography in the diagnosis of hepatocellular carcinoma and premalignant lesions in patients with cirrhosis. Hepatology 2001:34:1109-12

13 Schlottmann K, Klebl F, Zorger N, et al. Contrastenhanced ultrasound allows for interventions of hepatic lesions which are invisible on conventional B-mode. Z Gastroenterol 2004;42:303-10.

14 Colagrande S, La Villa G, Bartolucci M, et al. Spiral computed tomography versus ultrasound in the follow-up of cirrhotic patients previously treated for hepatocellular carcinoma: a prospective study. J Hepatol 2003:39:93-8.

15 Ho CL, Yu SCH, Yeung DWC. ${ }^{11} \mathrm{C}$-Acetate PET imaging in hepatocellular carcinoma and other liver masses. J Nucl Med 2003;44:213-21.

16 Caturelli E, Solmi L, Anti M, et al. Ultrasound guided fine needle biopsy of early hepatocellular carcinoma complicating liver cirrhosis: a multicentre study. Gut 2004;53:1356-62.
17 Schwerk WB Schmitz-Moormann P. Ultrasonically guided fine-needle biopsies in neoplastic liver disease: cytohistologic diagnoses and echo pattern of lesions. Cancer 1981:48:1469-77.

18 Sautereau D, Vire O, Cazes PY, et al. Value of sonographically guided fine needle aspiration biopsy in evaluating the liver with sonographic abnormalities. Gastroenterology 1987:93:715-18.

19 Brady PG, Goldschmid S, Chappel G, et al. A comparison of biopsy techniques in suspected focal liver disease. Gastrointest Endosc 1987;33:289-92.

20 Limberg B, Höpker WW, Kommerell B. Histologic differential diagnosis of focal liver lesions by ultrasonically guided fine needle biopsy. Gut 1987;28:237-41.

21 Sbolli G, Fornari F, Civardi G, et al. Role of ultrasound guided fine needle aspiration biopsy in the diagnosis of hepatocellular carcinoma. Gut 1990;31:1303-5.

22 Buscarini L, Fornari F, Bolondi L, et al. Ultrasound-guided fine-needle biopsy of focal liver lesions: techniques, diagnostic accuracy and complications. A retrospective study on 2091 biopsies. J Hepatol 1990;11:344-8.

23 Fornari F, Filice C, Rapaccini GL, et al. Small $(\leqslant 3 \mathrm{~cm})$ hepatic lesions. Results of sonographically guided fine-needle biopsy in 385 patients. Dig Dis Sci 1994;39:2267-75.

24 Caturelli E, Biasini E, Bartolucci F, et al. Diagnosis of hepatocellular carcinoma complicating liver cirrhosis: utility of repeat ultrasound-guided biopsy after unsuccessful first sampling. Cardiovasc Intervent Radiol 2002;25:295-9.

25 Atterbury CE, Enriquez RE, Desuto-Nagy Gl, et al. Comparison of the histologic and cytologic diagnosis of liver biopsies in hepatic cancer. Gastroenterology 1979;76:1352-7.

26 Giorgio A, Tarantino L, de Stefano G, et al. Complications after interventional sonography of focal liver lesions: a 22-year single-center experience. $J$ Ultrasound Med 2003;22:193-205.

27 Bret PM, Labadie M, Bretagnolle $M$, et al. Hepatocellular carcinoma: diagnosis by percutaneous fine needle biopsy. Gastrointest Radiol 1988; 13:253-5.

28 Roncalli M. Hepatocellular nodules in cirrhosis: focus on diagnostic criteria on liver biopsy. A Western experience. Liver Transpl 2004; 10:S9-15

29 Takamori R, Wong LL, Dang C, et al. Needletract implantation from hepatocellular cancer: is needle biopsy of the liver always necessary? Liver Transpl, 2000;6:67-72

30 Durand F, Regimbeau JM, Belghiti J, et al. Assessment of the benefits and risks of percutaneous biopsy before surgical resection of hepatocellular carcinoma. J Hepato 2001;35:254-8

31 Smith EH. Complications of percutaneous abdominal fine-needle biopsy. Radiology $1991 ; 178: 253-8$

32 Yamamoto J, Okada S, Shimada K, et al. Treatment strategy for small hepatocellular carcinoma: comparison of long-term results after percutaneous ethanol injection therapy and surgical resection. Hepatology 2001;34:707-13.

33 Bruix J, Sherman $M$, Llovet JM, et al. Clinical management of hepatocellular carcinoma. Conclusions of the Barcelona-2000 EASL conference. J Hepatol 2001;35:421-30.

34 Caturelli E, Ghittoni G, Roselli P, et al. Fine needle biopsy of focal liver lesions: the hepatologist's point of view. Liver Transpl 2004; 10:S26-9.

35 Yuen MF, Cheng CC, Lauder IJ, et al. Early detection of hepatocellular carcinoma increases the chance of treatment: Hong Kong experience. Hepatology 2000;31:330-5.

36 Bolondi L, Sofia S, Siringo S, et al. Surveillance programme of cirrhotic patients for early diagnosis and treatment of hepatocellula carcinoma: a cost effectiveness analysis. Gut 2001;48:251-9.

37 Mazzaferro V, Regalia E, Doci R, et al. Liver transplantation for the treatment of small hepatocellular carcinomas in patients with cirrhosis. N Engl J Med 1996;334:693-9. 